\begin{tabular}{|c|c|c|}
\hline 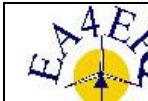 & $\begin{array}{c}\text { European Association for the } \\
\text { Development of Renewable Energies, Environment } \\
\text { and Power Quality (EA4EPQ) }\end{array}$ & $\begin{array}{l}\text { International Conference on Renewable Energies and Power Quality } \\
\text { (ICREPQ'12) } \\
\text { Santiago de Compostela (Spain), 28th to 30th March, } 2012\end{array}$ \\
\hline
\end{tabular}

\title{
Loss-Optimising-Management-Algorithm and the usage in an energy management for low-voltage grids
}

\author{
J. Teuscher ${ }^{1}$, V. Zeising ${ }^{1}$ and W. Schufft ${ }^{1}$ \\ ${ }^{1}$ Chair of Power Systems and High-Voltage Engineering \\ Faculty of Electrical Engineering and Information Technology \\ Chemnitz University of Technology \\ Straße der Nationen 62, 09107 Chemnitz (Germany) \\ Phone/Fax number:+0049 371531 37752/+0049 371531 837752, e-mail: jens.teuscher@etit.tu-chemnitz.de
}

\begin{abstract}
Since the amount of decentralised power generation is increasing it is important to develop an energy management system for low-voltage grids. This paper presents a method to operate such a management. The system is designed for managing a network of smart homes which can consume or supply electrical energy. The aims are to reduce the transmission losses and to stay within the limits of both, the voltage drop and the utilisation of lines and transformers. The reduction of the losses is implemented in the Loss-Optimising-ManagementAlgorithm (LOMA). This algorithm tries to find the power flow situation where minimal losses occur. The results of LOMA, the actual power situation (in low- and medium-voltage system) and the maximum power situation (based on the grid parameters) are summarised in an individual incentive signal for every smart home. The simulations show the feasibility of such an energy management and that significant loss reduction is achieved.
\end{abstract}

\section{Key words}

Smart grid, low-voltage grid, energy management, lossoptimisation

\section{Introduction}

There are more and more demands placed on low-voltage grids in recent years. On top of that the increase of renewable supplies and e-mobility creates additional challenges [1]. For example the grid operators in certain regions of Germany's south are forced to disconnect some photovoltaic systems because otherwise the voltage limits of the grid would be exceeded.

One option to meet the demands of an energy supply based on renewables is the implementation of a smart energy management system. This system manages the different storage systems and power supplies, it tries to achieve a load shifting. Such a smart management system is realised in two steps. The first step is a smart house which manages the different storage systems, power supplies and consumers in one house. The second step is the grid management which oversees the smart homes in one low-voltage grid segment and the connection to the medium-voltage grid. The focus of this paper is the grid management. Other grid management strategies focus on the utilization of the transformers and lines or on the voltage drop in low-voltage grids [2]. The main goal of this management is the reduction of losses in the lowvoltage grid, because the low-voltage grids are responsible for $75 \%$ of all losses on the whole way from a central power plant to the consumer [3]. The developed Loss-Optimising-Management-Algorithm (LOMA) minimises these losses. Its usage in an energy management system will be explained and the first simulation results for a typical urban low-voltage grid will be shown.

\section{Simulated low-voltage grid}

German low-voltage grids $\left(U_{r}=0.4 \mathrm{kV}\right)$ are connecting a number of households and small industries to the medium-voltage grid. There are differences between a typical urban and a typical rural low-voltage grid, which is shown in table I.

Table I. - Comparison between different low-voltage grids [4]

\begin{tabular}{|l|c|c|}
\hline & URBAN GRID & RURAL GRID \\
\hline $\begin{array}{l}\text { distribution trans- } \\
\text { former capacity } S_{r}\end{array}$ & $630 \mathrm{kVA}$ & $250 \mathrm{kVA}$ \\
\hline Max. line length & $200 \mathrm{~m}$ & $1400 \mathrm{~m}$ \\
\hline $\begin{array}{l}\text { Number of powered } \\
\text { households }\end{array}$ & 860 & 230 \\
\hline
\end{tabular}

The simulated low-voltage grids are based on these facts. In this paper the energy management is tested on a model of an urban low-voltage grid, which includes almost 80 connection points over an area of $0.25 \mathrm{~km}^{2}$. An average connection point supplies approximately 10 households and represents a described smart home. Connection points will be called nodes hereafter. Every node consumes 40 $\mathrm{kW}$ in maximum. This boundary condition is based on the typically used fuses. The maximum power supplied by each node is $10 \mathrm{~kW}$. This is based on a typical photovoltaic system occupying the average roof area or a typical combined heat and power plant for this number of households. 


\section{Development of the energy management}

\section{A. Loss-Optimising-Management-Algorithm}

The aim of the LOMA is to evaluate the consumers and suppliers in a grid based on the losses they affect. First it generates a sorted list of consumers and their losses in ascending order (final list). Its functionality is the same for the suppliers. To calculate the losses a power flow simulation is performed.

Fig. 1 shows a flow chart of the process. It is assumed that there are several consumers and suppliers in the grid.

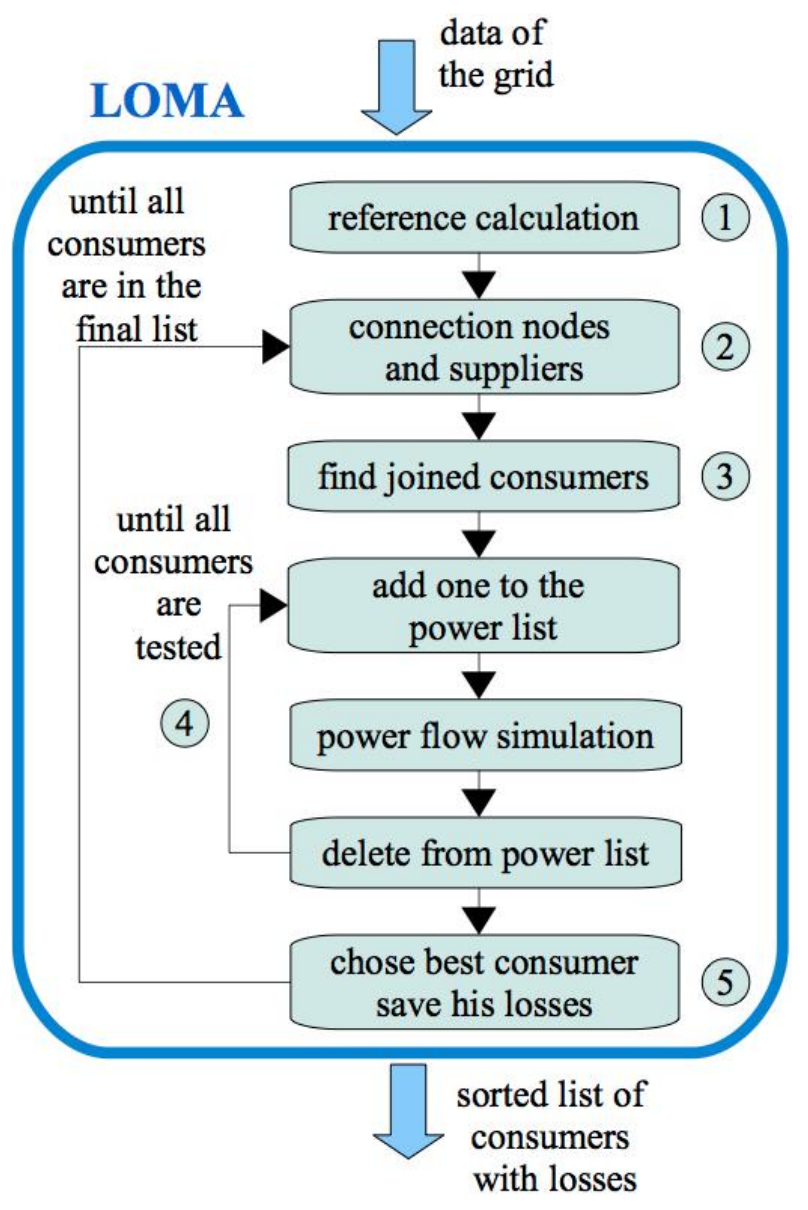

Fig. 1. Scheme of LOMA

(1) The first step is the calculation of the losses which occur when the energy of the suppliers flows back to the medium-voltage grid. For the power usage of all consumers is assumed to be zero. This is used as reference calculation because the resulting losses are the reference value in the incentive signal (chapter 3B).

Next an additional list (power list) is necessary. This will be the list with the nodes for the next power flow simulation. The generation node and the connected suppliers have to be appended to this list. The main iteration follows. It stops when all of the consumers are in the final list.

2 In a next step, the algorithm adds the connection nodes (these nodes are neither consumers nor suppliers) and the suppliers which are directly associated with one of the nodes in the power list.

3 As in the first step all consumers which are joined to one of the nodes in the power list have to be considered. The algorithm has to find the node that causes the smallest losses.

4 Thus, every consumer will be included in the power list separately. After determining the losses the consumer is deleted from the power list and the next one can be tested.

This cycle is repeated until all consumers which are connected to one of the nodes in the power list are tested with respect of their losses.

(5) Thereafter, the best node is chosen and its number and losses have to be saved in the final list. Furthermore, the number of the node has to be saved in the power list. After that it will not be tested again. The next loop of the iteration starts.

The functionality of the LOMA is shown on a small example grid illustrated in Fig. 2:

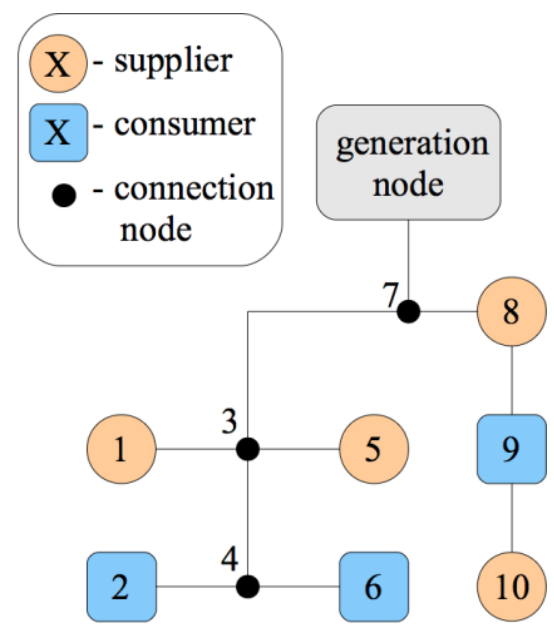

Fig. 2. Example grid

$\rightarrow$ The first step is the reference calculation with the generation node 0 and the suppliers $1,5,9$ and 10 (power of consumers 2, 6 and 9 is presumed as zero).

Table II. - Final list

\begin{tabular}{|c|c|}
\hline 0 (reference) & $P_{V, 0}$ \\
\hline
\end{tabular}

$\rightarrow$ Afterwards, the power list is applied and the connection nodes and suppliers which are associated with the generation node are added (step 2). These are the connection nodes 3, 4 and 7 and the suppliers 1,5 and 8 .

$$
\text { power list }=[0,3,4,7,1,5,8]
$$

$\rightarrow$ In a next step (3), the joined consumers 2, 6 and 9 have to be identified.

$\rightarrow$ Thereafter, the inner loop (4) is starting. It adds consumer 2 to the power list, computes the losses 
and deletes node 2 . The same procedure has to be done with the nodes 6 and 9 .

$\rightarrow$ In step 5 the consumer which effects the smallest losses (for example node 9) is chosen and the data is saved in the final list and the power list. power list $=[0,3,4,7,1,5,8,9]$

Table III. - Final list

\begin{tabular}{|c|c|}
\hline 0 (reference) & $P_{V, 0}$ \\
\hline 9 & $P_{V, 9}$ \\
\hline
\end{tabular}

$\rightarrow$ Due to the fact that there are consumers in the grid, which are not in the final list (nodes 2 and 6 ) a second run of the outer loop follows.

The algorithm starts again with step 2 and adds supplier 10 to the power list (because it is joined with consumer 9 which is in the power list now). power list $=[0,3,4,7,1,5,8,9,10]$

$\rightarrow$ The inner loop is starting again. The consumers 2 and 6 are tested again. The node with the smallest losses is selected (for example 2). The last step of the inner loop is to save the chosen node in the power list and in the final list:

power list $=[0,3,4,7,1,5,8,9,10,2]$

Table IV. - Final list

\begin{tabular}{|c|c|}
\hline 0 (reference) & $P_{V, 0}$ \\
\hline 9 & $P_{V, 9}$ \\
\hline 2 & $P_{V, 2}$ \\
\hline
\end{tabular}

$\rightarrow$ As well as before the outer loop runs. Step 2 is done again but there are no further connection nodes or suppliers. The power list does not change.

$\rightarrow$ In step 3 only node 6 is found (because it is the last consumer). Thus, this node is the only one which has to be tested. Hence, it is automatically the best node and is saved in the power list power list $=[0,3,4,7,1,5,8,9,10,2,6]$

Table V. - Final list

\begin{tabular}{|c|c|}
\hline 0 (reference) & $P_{V, 0}$ \\
\hline 8 & $P_{V, 8}$ \\
\hline 2 & $P_{V, 2}$ \\
\hline 6 & $P_{V, 6}$ \\
\hline
\end{tabular}

$\rightarrow$ Due to the fact that all existing consumers in the grid are in the final list the outer loop stops now and the algorithm is finished.

The procedure for generating the final list for the suppliers is the same. The only difference is the interchanging role of suppliers and consumers in the explanation above.

The practical implementation is based upon some aspects of graph theory, for example the definition of a special adjacency matrix [5].

\section{B. Energy management based on an incentive signal}

The LOMA is only one part of the whole energy management. Fig. 3 gives an overview of the management system. It combines four terms to compute an incentive signal for each individual node [6].

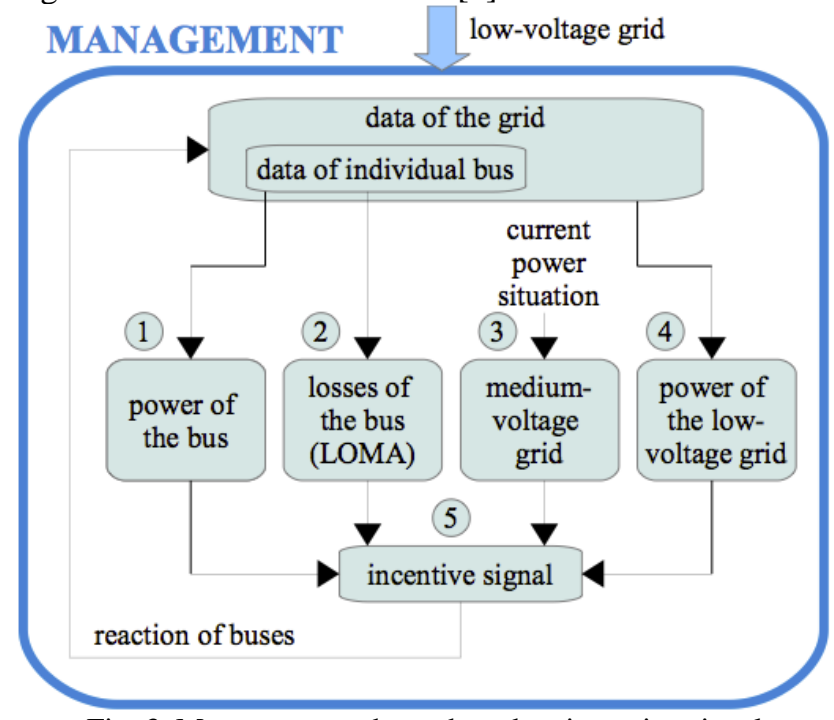

Fig. 3. Management scheme based on incentive signal

1 The first term analyses the individual power situation of the nodes in comparison with the maximum of possible power in the whole grid.

2 One of the main goals is to reduce losses. This leads to the second term in the management. To evaluate a node by its losses the final list which is generated by the LOMA is used.

The incentive signal [6] uses the differences of saved losses between two consecutive consumers in the final list. The reference calculation is necessary to determine the difference to the first node in the final list. The differences for the signal in the case of the example of chapter 3.1 would look as follows.

Table VI. - Differences

\begin{tabular}{|c|c|}
\hline 8 & $P_{V, 8}-P_{V, 0}$ \\
\hline 2 & $P_{V, 2} \cdot P_{V, 8}$ \\
\hline 6 & $P_{V, 6} \cdot P_{V, 2}$ \\
\hline
\end{tabular}

If the difference is negative, the node reduces the losses in the grid. This case appears if there are many suppliers in the grid. In such a situation more losses arise if the whole power flows back to the medium-voltage grid, than if it flows to a consumer in the grid (less length of lines). In the case of a positive difference the consumer increases the resulting losses in the grid. The same differences have to be computed for the final list of suppliers.

3 The third element comprises the current power situation in the medium-voltage grid in relation to the maximum permissible power. This term is necessary for handling peaks of wind energy or other surplus in the medium-voltage grid. If a wind-energy peak occurs, the consumers should consume more electrical energy. 


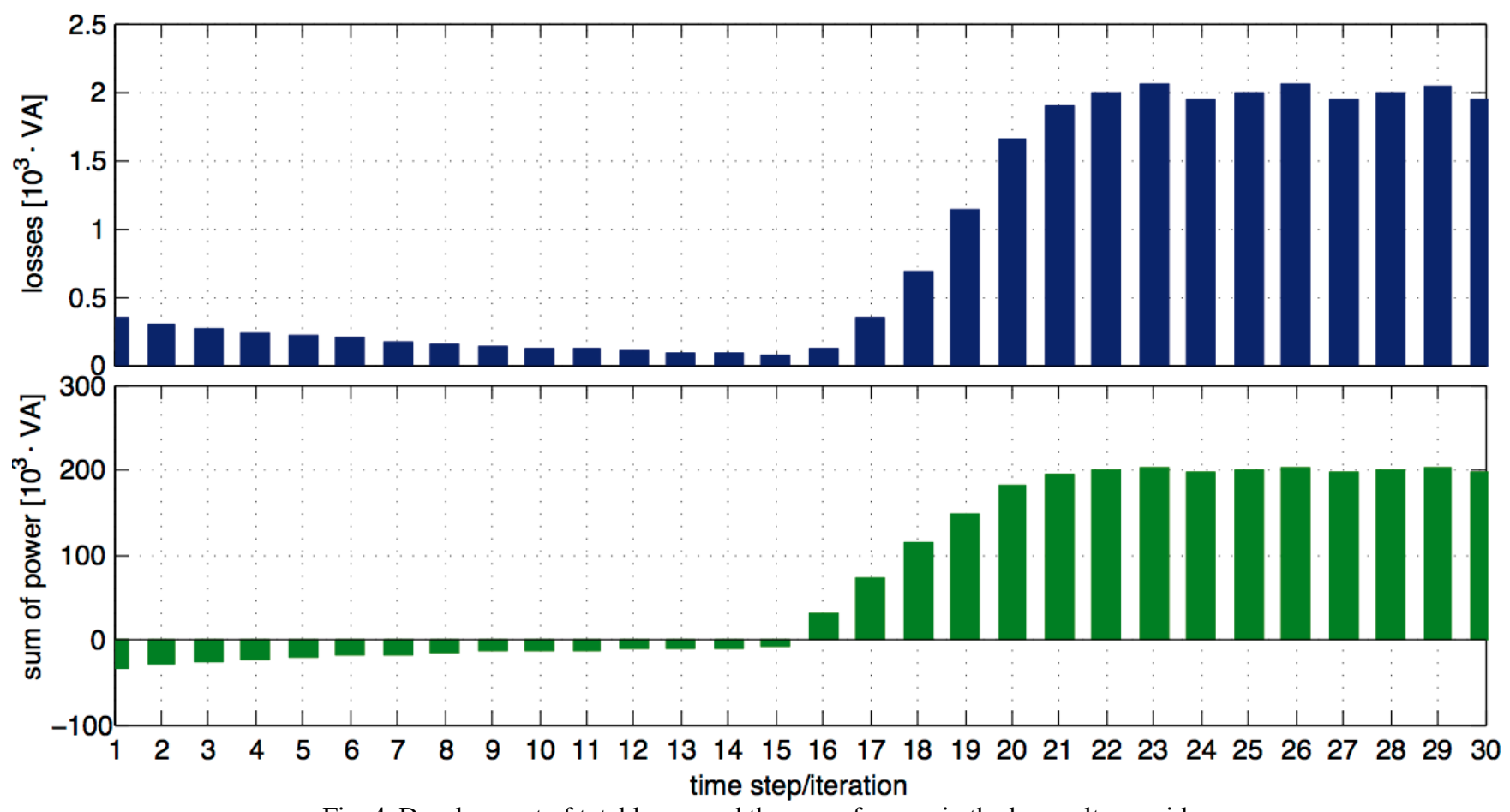

Fig. 4. Development of total losses and the sum of power in the low-voltage grid

In this situation term three of the incentive signal is positive.

4 To stay within limits of the low-voltage grid (voltage drop, utilisation of lines and transformer) the fourth term in Fig. 3 was included. . Thus, it depends on the power in the whole low-voltage grid.

The final incentive signal is calculated from the four terms. All four terms are weighted with individual factors. The factors can be adjusted to change the characteristic of the incentive signal based on the desired behaviour of the low-voltage grid. For example, if there is a surplus of wind energy, it could be more important spending this energy than reducing the losses.

In general, if the signal for one node is positive it is convenient for this node to consume more or supply less depending on its current situation. On the other hand a negative signal requires a reduction of consumption or accordingly an increasing of generation.

\section{Results}

\section{A. Simulation setting}

For testing the final incentive signal a model of a typical urban low-voltage grid is used. At the beginning the current power situation is random and the power flow from the medium-voltage grid is assumed to be zero. After 15 time steps the power in the medium-voltage grid increases up to $200 \mathrm{kVA}$.

Every time step has an individual length of time and describes the time from one change of the power-situation to another one.

We assume that all nodes react to the incentive signal. A stochastic non-reaction is also implemented but would not be significant to show the robustness of the management.
A stochastic non-reaction of nodes induces erratic figures and the traceability of the management would be much more difficult.

\section{B. Reaction of the grid}

The first 15 time steps the low-voltage grid has to be independent. If the sum of power in the whole grid becomes zero the suppliers supply the consumers in the grid. In Fig. 4 the development of total losses and the sum of power in the low-voltage grid is shown. The signal enforces a reduction of losses and the convergence of the sum of power to zero. The sum of the absolute of the power of all nodes (power flow in the grid) remains constant. Thus it can be concluded: the incentive signal provokes a beneficial distribution of the power so that the same power flows with fewer losses. During the first 15 time steps the incentive signal does not change its sign but becomes smaller. This proves: if the system is not interfered with it, it converges to a stable and independent state.

In Fig. 5 the development of the incentive signal and the corresponding power of four exemplary nodes are illustrated. Fig. 5 shows the change of the incentive signal to a high positive level after the 15 iterations to balance the change in the medium-voltage grid. At time step 21 the sum of the power of all nodes achieves the given value of the medium-voltage grid. Therefore the incentive signal decreases to a low level. Meanwhile, as is visible in Fig. 4 the power of the nodes themself stays constant for the following iterations.

\section{Discussion}

The main advantage of the management is the amount of degrees of freedom for the user. With the help of the weighting coefficients it is possible to control the behaviour of the management. On the other hand the long 


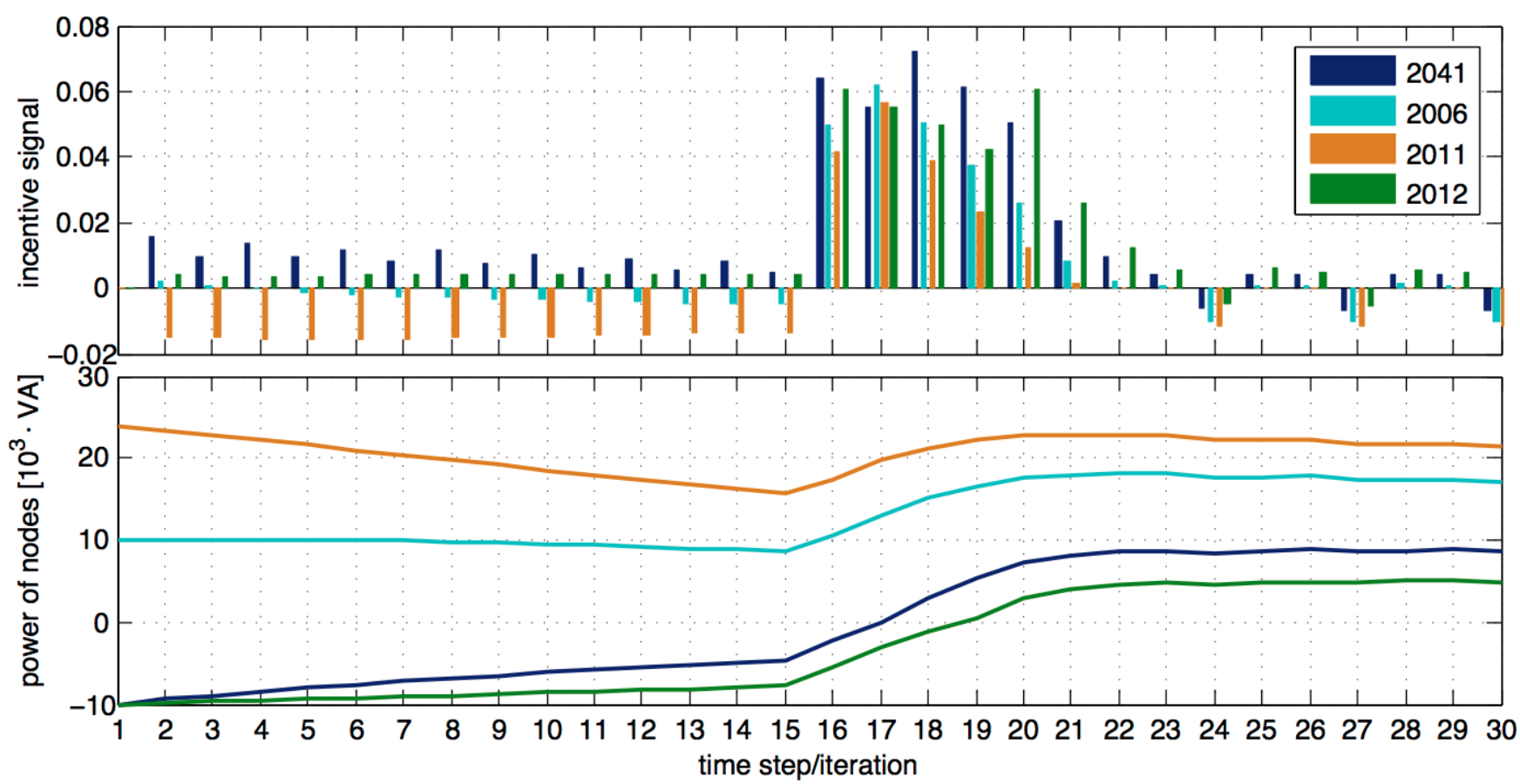

Fig. 5. Development of incentive signal and the power of four exemplary nodes

calculation time for the power-flow simulation is a big drawback. Thus the next step is to discard the power-flow simulations or at least to reduce the number of necessary power-flow simulations.

Another important fact is that only known losses can be reduced with the management system. Real low-voltage grids are constructed with a high number of sleeves between different cables, terminals, fuses and other grid equipment. All these utilities create unknown losses which are not observed in the power-flow simulation. The reduction of the losses with the management system is only based on the transmission losses of the lines. But in the majority of situations this reduction minimise the power flow way. In the case of a short power-flow-way the number of such utilities with unknown losses is in most situations minimised. Thus the management system is also working expedient with regard to the unknown losses.

\section{Conclusion}

This paper describes an energy management based on an incentive signal with the aid of a new algorithm to reduce losses. The energy management combines the load situation in the medium-voltage grid and the distribution of consumers and suppliers. It is able to transform this information into an interpretable incentive signal. With the help of LOMA losses can be reduced and overload in the grid can be avoided. The results show the stability and the effective operation of the management. A power peak in the medium-voltage grid can be dealt with, be it positive or negative. And even if a node does not react to the incentive signal the surrounding nodes compensate its behaviour.
It is established that it is possible to create a management which reduces losses and regulates consumer, supplier and their positions to each other.

\section{Acknowledgement}

This work is one part of the research group smart distributed power-storage systems and is supported by the European Social Fund.

\section{References}

[1] ETG Task Force Smart Metering: "Smart Energy 2020 Vom Smart Metering zum Smart Grid", VDE, Frankfurt, 2010

[2] I. Wasiak, M. C. Thoma, C.E.T. Foote, R. Mienski, R. Pawelek, P. Gburczyk, G.M. Burt: "A Power-Quality Management Alorithm for Low-Voltage Grids With Distributed Resources, IEEE Transactions on Power Delivery, Vol. 23, No. 2, 2008

[3] K. Mehlhorn, J. Kliemt: "Bestimmung der elektrischen Verluste im Netz eines städtischen Netzbetreibers", ew Jg. 103 (2004), H1-2, S.20-23

[4] J. Teuscher, A. Götz, W. Schufft: "Electric vehicles and their effects in low-voltage grids“, ICREPQ'11. Las Palmas de Gran Canaria, 2011

[5] R. K. Ahuja, T. L. Magnanti, J. B. Orlin: "Network flows - theory, algorithms and applications", Prentice Hall, 1993

[6] J. Teuscher, D. Kühnert, V. Zeising: "Loss-optimized management in low-voltage grids based on an incentive signal“, University of West Bohemia, Plzen, 2011 